\title{
A ZIGBEE AND EMBEDDED BASED SECURITY MONITORING AND CONTROL SYSTEM
}

\author{
S. Kanagamalliga ${ }^{1}$, Dr. S. Vasuki ${ }^{2}$, A. Vishnu Priya ${ }^{3}$, V. Viji ${ }^{4}$ \\ ${ }^{1}$ Assistant Professor, ${ }^{2}$ Professor and Head, ${ }^{3,4,5}$ U.G Student \\ Department of Electronics and Communication EngineeringVelammal College of \\ Engineering and Technology, Madurai
}

\begin{abstract}
As technology becomes more advanced and modernized; more features are added to the existing system for the purpose of satisfying the increasing security needs of the people. Deploying wireless technologies for security and control in home offers attractive benefits along with user friendly interface. In this paper we present a smart security system comprises of Zigbee, GSM, Sensors and Smartphone for Security monitoring and control, when the user is at remote premises. Three sensors namely $P$

$I R$, vibration and door sensor are installed at windows and doors. Whenever there is a security breach the sensors sends the signal to the Pic microcontroller. The Max 232 converter provides serial interface between the microcontroller and the zigbee. The Zigbee end device then transmits the signal to the Zigbee coordinator. The ZC at the control console communicates with MCU using max 232 converters. MCU would in turn access and control the GSM module via AT commands and automatically sends SMS to the owner's mobile phone informing him about the security breach. On receiving the SMS, user can make a video call using Skype account; Smartphone installed at home will feed us with a live video. On viewing the video the user can know whether an intruder has entered or not. Then accordingly faint gas valve and door lock can be controlled by the user. Thus whenever there is an intrusion, our system lets the user to monitor and control his premises from any part of the world.
\end{abstract}

\section{Keywords}

ZC (Zigbee Coordinator), MCU (Micro Controller), GSM (Global System for Mobile Communication).

\section{INTRODUCTION:}

A Security system is considered to be best only if it offers protection and monitoring that guard against a number of threats, protecting homes against the elements as well as break-in and home invasions. A security system should be customizable, provide excellent monitoring and allow communication with the owner. During recent past, a number of systems were introduced for security measurements based on wired networks. In literature, researchers suggested a number of security systems based on new technologies like GSM (Global System for Mobile communication), USN (ubiquitous sensors network) and implemented through FPGA (field programmable gate arrays), DSP (digital signal processor), and MCU (microcontroller unit). [1] developed a Java based home automation system. An embedded board physically connected all the home automation devices and, through integration with a personal computer (PC) based web server, provided remote access to the system. The use of Java technology, which incorporates built-in network security features, produces a secure solution. However, the system requires an intrusive and expensive wired installation and the use of a high end PC. [2] introduced a 
Bluetooth based home automation system, consisting of a primary controller and a number of Bluetooth sub-controllers. Each home device is physically connected to a local Bluetooth subcontroller. The home devices communicate with their respective sub-controller using wired communications. From the sub-controller all communications are sent to the primary controller using wireless communications. It is desirable for each home device to have a dedicated Bluetooth module. However, due to the fiscal expense of Bluetooth technology, a single module is shared amongst several devices. This architecture reduces the amount of physical wiring required and hence the intrusiveness of the installation, through the use of wireless technology. However, the architecture does not completely alleviate the intrusiveness of the installation due to the incorporation of some wired communications. Moreover the sharing of a single Bluetooth module between numerous devices has the disadvantage of incurring an access delay. [3] Describes the architecture and simulation of a GSM based remote sensing and control system using FPGA. [4] Explains home automation system using GSM, Internet and speech recognition. In this system the home gateway is internet which require personal computer (PC). However, it's hard to manage PC and keep it $\mathrm{ON}$ all the time. Also it consumes more power. The system presented in [5] is an internet-based intelligent system for home power management aiming to reduce energy consumption. This system also uses internet cloud as a home gateway having the same limitations as described earlier. In [6] authors proposed a zigbee based home network configuration. This system controls all home appliances through zigbee-infrared combination and zigbee power adapter.

\section{PROPOSED SYSTEM}

The project can be splitted into two parts: the hardware part and the software part. The first part deals about incorporating MCU with sensors, ZigBee architecture, and mobile phone to build a working circuit that supports portability without compromising reliability, and the second part is to make an ingenious $\mathrm{C}$-code for programming the $\mathrm{MCU}$ to monitor and control secured environment.

\subsection{HARDWARE}

The system hardware consists of ZigBee EM357module, Pic micro controller, GSM module, a smart phone with net connectivity and corresponding interfaces. Zigbee is a widely deployed standard for providing communication between sensors in low data rate wireless networks. They are low cost, low power digital radios for energy efficient and cost effective intelligent devices. It is based on IEEE 802.15 Wireless Personal Area Networks standard. It operates in the frequency range of $2.4 \mathrm{GHZ}$ ISM band and has a data rate of $250 \mathrm{kbps}$. The transmission distances range from 10 to 100 meters line-of-sight, depending on power output and environmental characteristics. Zigbee devices can form networks with Mesh, Star and Generic Mesh topologies among themselves. The network can be expanded as a cluster of smaller networks. A ZigBee network can have three types of nodes: Zigbee Coordinator (ZBC), Zigbee router (ZBR) and Zigbee End Device (ZBE) each having some unique property. In a network there is only one Coordinator which acts as the main control panel and stores information about the network. Any end device like lighting units, air conditioning elements etc. can be Zigbee End Devices. They communicate only with the coordinator and that is the point where sensors are deployed. A ZBR is an optional component used to extend the coverage. There are two modes of data transfer namely Beacon mode and Non Beacon mode. In Beacon mode, when the devices are not sending the data they may enter a low power state and reduces the power consumption. In Non-beacon mode, the end devices need to be wake up only while sending the data while the routers and coordinators need to be active most of the time. 


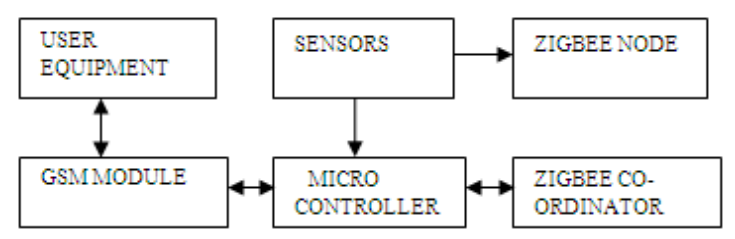

Figure 1. Block Diagram

The Zigbee module can be installed with any sensor and home appliances as it is very small in size i.e. $21 \mathrm{~mm} \times 37 \mathrm{~mm}$, and a low power consuming device. In the design three sensors namely PIR sensor, vibration sensor and magnetic door contact sensor are used. While the PIR sensor is used for detecting human presence, the vibration sensor and magnetic door contact sensor installed at the windows and doors are used for detecting window breaking and door opening. ZED is connected with door contact sensor, PIR sensor, and door lock system respectively. ZigBee node continuously checks input from the corresponding sensor and sends a wireless message to the $\mathrm{Zc}$.

The control console which consists of Pic micro controller and ZC CS2520 module that are mounted on same PCB in our design. ZC is interfaced with MCU using max 232 converters, thus giving information to $\mathrm{MCU}$ about sensors status. Also $\mathrm{ZC}$ takes instructions from $\mathrm{MCU}$ for actuators using the same interface. MCU is heart of the system. On one hand it is interfaced with ZC while on the other hand it is communicating with the GSM module. Mobile phone can be accessed through its ports using corresponding serial cable. There occurs a serial communication between MCU and mobile phone. To allow compatibility of data communication between MCU and mobile phone, an interfacing standard RS-232 was employed. And for this purpose MAX232 level converter IC is used. Finally, the GSM module is wirelessly connected with the user mobile phone through GSM network. GSM module consists of a GSM modem assembled together with power supply circuit and communication interface max 232 converters for micro controller. GSM modem is a device that generates, transmit or decode data from a cellular network, for establishing communication between the cellular network and the micro controller. DC Motors convert electrical energy (voltage or power source) to mechanical energy (produce rotational motion). They run on direct current. The Dc motor works on the principle of Lorentz force which states that when a wire carrying current is placed in a region having magnetic field, than the wire experiences a force. When we pass the input DC current to the coil through the brushes, it directly goes to the coil inside the motor body. This makes coil to work as an electromagnet. Magnetic fields of both magnets interact with each other that results in a force which in turn produces the necessary torque required to move the coil. This torque drives the coil to move round and a shaft attached with the coil moves too. A gas spray is placed in contact with the shaft. When the DC motor works, the shaft exerts a force on the nozzle of the gas spray and as a result the gas gets sprayed. Door opening and closing is controlled by means of a relay.

\subsection{SOFTWARE}

The ZigBee module CS2520 is supported by insight desktop a comprehensive integrated development environment (IDE) and C-Language compiler tool-chain. Ember insight desktop is designed for writing, compiling, loading and debugging software on to multiple nodes in a networked environment. Now to connect this chip with the sensors and MCU, max 232 converter is deployed. The sensor node EM357 uses a built-in 32-bit ARM Cortex-M3 microprocessor to control all the interfaces and uses RF antenna at the end for communication purpose. The 
microprocessor is programmed via an ISP header on board to program flash memory of the chip. On the other hand Pic microcontroller is a general purpose MCU with a rich set of built-in peripherals. The MCU also features a built-in Flash and EEPROM memories. The flash is used to store code that is also programmed via the ISP header on the board. For the proposed system, the $\mathrm{MCU}$ on one hand deals with the $\mathrm{ZC}$ to receive intrusion detection from the sensors installed while on the other hand it deals with the GSM module to send Short Message Service (SMS) or to call the owner. For that purpose searching through answer comes out to be the max 232 converter for interfaces. Interfacing standards used in this work are summarized in Table 1.

Table 1. Interfacing standards

\begin{tabular}{|l|l|}
\hline DEVICES & INTERFACE \\
\hline Sensors $\leftrightarrow$ MCU & EM357 UART \\
\hline MCU $\leftrightarrow$ ZED & Max 232 \\
\hline ZED $\leftrightarrow$ ZC & Zigbee RF Communication \\
\hline ZC $\leftrightarrow$ MCU & Max 232 \\
\hline MCU $\leftrightarrow$ Mobile phone & RS232 \\
\hline Mobile Phone $\leftrightarrow$ User mobile phone & GSM Communication \\
\hline
\end{tabular}

Now that it is all set for the communication between mobile phone and the MCU, in order to control the mobile phone through its ports, we used AT Commands, provided by the vendor companies. AT commands are used to control MODEMs. AT is the abbreviation for Attention. These commands come from Hayes commands that were used by the Hayes smart modems. The Hayes commands started with AT to indicate the attention from the MODEM. The dial up and wireless MODEMs (devices that involve machine to machine communication) need AT commands to interact with a micro controller. These include the Hayes command set as a subset, along with other extended AT commands. AT commands are used to access and control the built in modem of the mobile phone. Table 2 shows AT -commands that are used in the proposed system.

Table 2. AT Commands

\begin{tabular}{|l|l|}
\hline COMMANDS & DESCRIPTION \\
\hline ATD & Call dialing \\
\hline AT + CMGS & SMS to specific number \\
\hline AT + CMGD & Delete unauthorized SMS \\
\hline AT + CMGR & Read authorized SMS \\
\hline AT + CMEE & $\begin{array}{l}\text { To report mobile equipment } \\
\text { error }\end{array}$ \\
\hline
\end{tabular}

\section{OPERATION}

When the user is at remote premises and if there is a security breach then he is informed about it through SMS. The user in turn can control the gas valve and door lock through the reply SMS. The working of the security monitoring and control system is as follows. Three sensors namely PIR, vibration and magnetic read switch are used. Vibration and magnetic read switch are installed at doors and windows. PIR sensor is sensitive to the infrared radiation emitted by human being and hence used for human detection. Vibration sensor is used for monitoring vibration and hence used for detecting window breaking. Magnetic read switch is used for sensing door 
breaking or door opening. When the intrusion is detected by these sensors, it then sends the signal immediately to the microcontroller. The micro controller converts the analog signals in to digital signals for transmitting it to the zigbee. The zigbee is interfaced with the microcontroller using the max 232 converter which provides serial communication. The Zigbee end device then transmits the signal to the zigbee coordinator. The $\mathrm{ZC}$ is interfaced with the microcontroller by means of max 232 converter. A GSM/GPRS module consists of a GSM modem with standard communication interface max 232 converter, so that it can be easily interfaced with a microcontroller. The power supply circuit is also built in the module that can be activated by using a suitable adaptor. AT commands are sent by the microcontroller to the GSM module for communication with the GSM cellular network. Once intrusion is detected, a text message is sent to the owner indicating security breach. On receiving the text message, the user can use the Skype account in the mobile phone for making a video call. The Smartphone with net connectivity, present in the home feeds the owner with a live video. On viewing the video the user can know whether the intruder is a known person or not. Then the user can communicate with the system through reply SMS. If there is a reply SMS from the user, the GSM module communicates with MCU in the same manner, but in reverse direction. The mobile phone number is first scanned, if it is authorized, then further communication is made. Then accordingly the owner can control the faint gas valve and lock the door. The faint gas valve is activated by SMS sent by the owner, the DC motor starts working. The shaft of the DC motor exerts a force on the gas sprayer and as a result the gas starts spraying throughout the room. Then finally the user can lock the door by sending the corresponding command to the GSM module. Thus the system is not only used for monitoring but also for controlling purpose ensuring the reliability and safety of home.

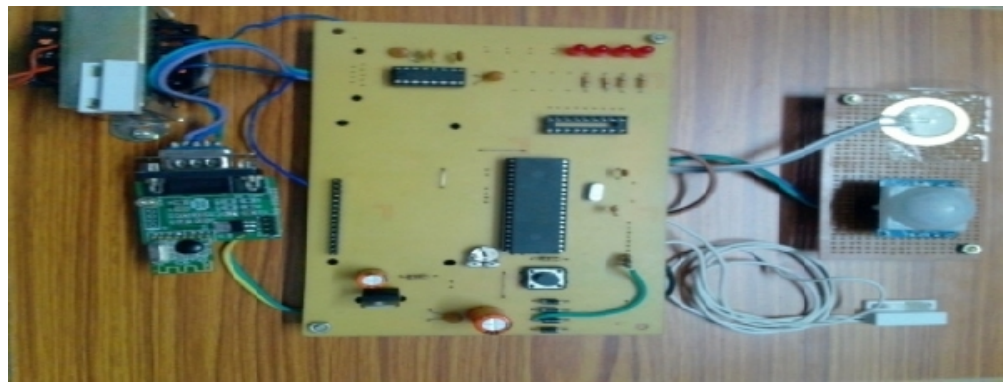

Figure 2. Intrusion Detection

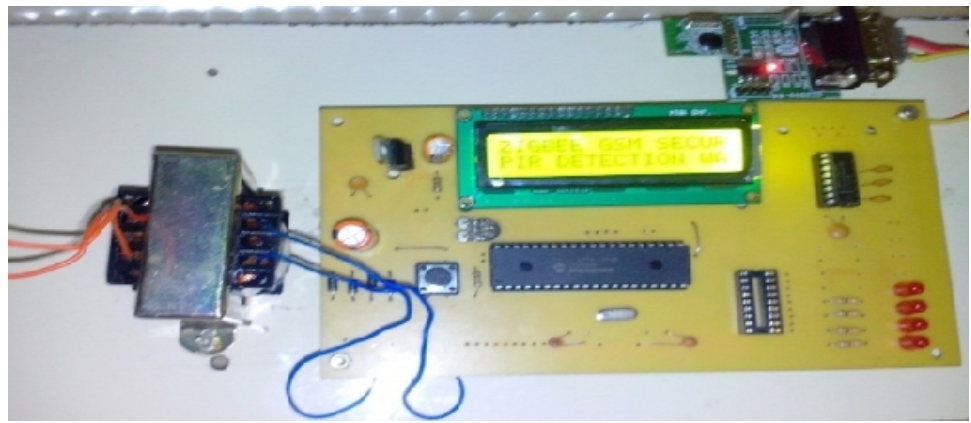

Figure 3. LCD display showing intrusion detection 


\section{CONCLUSION}

A novel architecture for a smart security system is proposed and implemented, using the wireless communication technology ZigBee, wide covered GSM and app installed Smartphone. MCU is programmed for the system and is the heart of our project. The use of ZigBee communication technology helps lower the expense of the system and the intrusiveness of the respective system installation. The GSM communication provides worldwide coverage, easy and a low cost way of information interchange. The MCU provides interoperability of ZigBee and GSM and also makes this system portable and easy to install as the system is embedded. The system is designed to provide a secure environment by letting the user know about the security breach and who is involved in it. Not only that it also allows the user to control gas valve and door lock. Thus it enables the remote user to monitor and control his premises from any part of the world.

\section{REFERENCES}

[1] A. R. Al-Ali and M. Al-Rousan, "Java-based home automation system", IEEE Transactions on Consumer Electronics, vol. 50, no. 2, pp. 498-504, 2004.

[2] N. Sriskanthan, F. Tan and A. Karande, "Bluetooth based home automation system", Microprocessors and Microsystems, Vol. 26, no. 6,pp. 281-289, 2002.

[3] Wael M EI-Medany, Mahmoud R EI-Sabry. "GSM-Based Remote Sensing and Control System using FPGA" Proceedings of the International Conference on Computer and Communication Engineering 2008(ICCCE08) .

[4] Baris Yuksekkaya, A. Alper Kayalar, M. Bilgehan Tosun, M. Kaan Ozcan, and Ali Ziya Alkar. "A GSM, Internet and Speech Controlled Wireless Interactive Home Automation System" IEEE Transactions on Consumer Electronics, Vol.52 No. 3, and pp: 837-843, 2006.

[5] Manouchehr Ghahramanian Golzar, HamidReza Tajozzakerin. "A New Intelligent Remote Control System for Home Automation and Reduce Energy Consumption" Fourth Asia International Conference on Mathematical/Analytical Modeling and Computer Simulation. pp 174-180, 2010.

[6] II-Kyu Hwang, Member, IEEE and Dae-Sung Lee, Jin-Wook Baek. "Home Network Configuring Scheme for All Electric Appliances Using ZigBee-based Integrated Remote Controller" IEEE Transactions on Consumer Electronics, Vol. 55, No. 3,pp 1300-1307, August 2009.

[7] Khusvinder Gill, Shuang-Hua Yang, Fang Yao, and Xin LuA "ZigBee-Based Home Automation System” IEEE Transactions on Consumer Electronics, Vol. 55, No. 2, MAY 2009.

[8] Xiaohua Zeng, Abraham O. Fapojuwo, and Robert J. Davies, "Design and Performance Evaluation of Voice Activated Wireless Home Devices" IEEE Transactions on Consumer Electronics, Vol. 52, No. 3, AUGUST 2006. 